\title{
Kinetic, kinematic, magnetic resonance and owner evaluation of dogs before and after the amputation of a hind limb
}

\author{
Vladimir Galindo-Zamora ${ }^{1,2}$, Verena von Babo ${ }^{1}$, Nina Eberle ${ }^{1}$, Daniela Betz ${ }^{1}$, Ingo Nolte ${ }^{1 *}$ and Patrick Wefstaedt ${ }^{1}$
}

\begin{abstract}
Background: The amputation of a limb is a surgical procedure that is regularly performed in small animal practice. In spite of several clinical reports indicating high owner satisfaction after limb amputation in dogs, an amputation is still very critically seen by the owners, and even by some veterinarians, due to the lack of accurate information about the recovery of amputee patients. Thus, the objective of this study was to prospectively evaluate, both objectively and subjectively, the recovery outcome of dogs undergoing a hind limb amputation. Twelve patients in which a hind limb amputation was scheduled were studied. Kinetic and kinematic gait analyses were performed before the amputation, and 10,30, 90 and 120 days after surgery. Magnetic resonance (MR) examination of the contralateral stifle joint was performed before and 120 days after amputation. The subjective impressions of the owners were gathered at the same examination times of the gait analyses.

Results: Kinetic data showed a redistribution of the load to all remaining limbs after the amputation; ten days after the procedure patients had already established their new locomotory pattern. Kinematic data showed significant differences between sessions in the mean angle progression curves of almost all analyzed joints; however, the ranges of motion were very similar before and after the amputation, and remained constant in the subsequent sessions after the amputation. No changes in the signal intensity of the soft tissues evaluated, and no evidence of cartilage damage or osteoarthritis was seen on the MR examination of the contralateral stifle. Owners evaluated the results of the amputation very positively, both during and at the end of the study.

Conclusions: Dogs had a quick adaptation after a hind limb amputation, and the adaptation process began before the amputation was performed. This happened without evidence of morphologic changes in the contralateral stifle joint, and with a very positive evaluation from the owner.
\end{abstract}

Keywords: Hind limb amputation, Kinetic and kinematic analyses, Magnetic resonance imaging, Owner evaluation

\section{Background}

The amputation of a limb is a surgical procedure that is regularly performed in small animal practice. Severe trauma and limb tumors are the most common reasons for performing an amputation; other indications include chronic osteomyelitis, neurological dysfunctions such as sciatic neuropathy and brachial plexus paralysis, congenital

\footnotetext{
* Correspondence: ingo.nolte@tiho-hannover.de

The preliminary results of this study were presented at the 56 .

Jahreskongress der Deutsche Gesellschaft für Kleintiermedizin on October 22,

2010 in Düsseldorf (Germany).

'Small Animal Hospital, University of Veterinary Medicine Hannover,

Foundation, Bünteweg 9, D-30559 Hannover, Germany

Full list of author information is available at the end of the article
}

limb deformities, vascular disease and arteriovenous fistulas [1-3].

In spite of several clinical reports indicating high owner satisfaction after limb amputation in dogs [4-7], an amputation is still very critically seen by the owners, and even by some veterinarians. Particularly, owners have the tendency to think that the procedure may affect the animals emotionally, as it indeed happens in people [8], or that it will be disabling for them. Besides, owners are often worried about the possibility of overload of the remaining limbs, leading to hypothetical secondary joint pathologies. Thus, many owners are reluctant to have their dog amputated and reject the amputation as an alternative to euthanasia or take the decision only after 
the patient has gone through a painful surgical and/or medical treatment process.

The lack of objective information prevents the veterinarian from providing the owners with accurate information about these concerns. A hesitant veterinarian might then play a role in the owner deciding against the amputation. There is only one previously published report objectively evaluating the gait of amputated dogs [9]. In that study, force plate analyses were carried out to measure ground reaction forces (GRF) and contact times in a population of 10 large-breed dogs which had a limb amputation (five forelimbs and five hind limbs). Additionally, the center of gravity was calculated in those patients. It was found that the absence of a limb caused statistically significant changes in the GRF, impulses and contact times of the remaining limbs and the location of the animal's centre of gravity, in comparison to a control group of 22 healthy dogs. However, there are no prospective studies with animals which are planned to be amputated, and no study has been performed objectively evaluating kinematics (joint movement) or possible joint changes after a hind limb amputation in dogs.

The general objective of the present study was therefore to prospectively characterize the recovery outcome of dogs undergoing a hind limb amputation. In order to evaluate the motion and weight bearing characteristics, as well as the duration of adaptation to the three-legged gait, kinematic and kinetic analyses were carried out. Furthermore, MR images of the remaining contralateral femorotibial (stifle) joint were made before and 4 months after the amputation, in order to investigate possible changes in joint morphology, due to a hypothetic weight bearing overload of this limb.

It was hypothesized that there would be marked changes both in the kinetic and the kinematic parameters after the amputation, but that those changes would not impair the ability of the animal to lead a normal life. Based on our clinical experience and some of the aforementioned studies [4-7], it was also hypothesized that there would not be any changes in the contralateral stifle on the MR examination. Thus, after the initial reluctance to the amputation, owners would be satisfied with the procedure.

\section{Methods}

This study was carried out in accordance with the German Animal Welfare Guidelines and was approved by the Ethics Committee of the Lower Saxony State Office for Consumer Protection and Food Safety (Approval Number: 10A071). All owners agreed to their dogs participation in the study and signed a consent form.

\section{Patients}

All dogs presented to the Small Animal Hospital of the University of Veterinary Medicine Hannover, Foundation
(Germany), between March 2010 and October 2011, for a hind limb amputation were included in the study. In total, 12 patients were enrolled. Two additional patients were not included due to aggressiveness in one case, and presence of metallic orthopedic implants in both stifle joints, making it unadvisable to perform the MR examination, in the other case.

Before surgery a thorough physical examination, including an orthopedic and neurologic examination of all remaining limbs and the spine, was performed to rule out any disease which might obscure the results. This examination was repeated 10, 30, 90 and 120 days after the amputation. It was planned that, in case an abnormality was suspected, all necessary diagnostic examination tools would be used to determine the type and location of such an abnormality and its possible relationship with the amputation.

\section{Surgical procedure}

On the amputation day, physical status was determined based on the physical examination, blood work and other diagnostic tests as needed. Based on the American Society of Anesthesiologists (ASA) physical status classification system, all patients were classified as ASA 2 (patients with local or mild systemic disease). The animals were premedicated using a combination of levomethadone $(0.6 \mathrm{mg} / \mathrm{kg})^{1}$ and diazepam $(0.5 \mathrm{mg} / \mathrm{kg})^{2}$; anesthesia was induced with propofol dosed to effect (1$4 \mathrm{mg} / \mathrm{kg})^{3}$ After orotracheal intubation, anesthesia was maintained with isoflurane ${ }^{4}$ in a 1:1 oxygen: air mixture adjusted according to the physical signs of anesthetic depth (end-tidal isoflurane 0.7-1.5 vol \%) and a continuous rate infusion (CRI) of fentanyl $(0.16 \mu \mathrm{g} / \mathrm{kg} / \mathrm{min}){ }^{5}$ lidocaine $(50 \mu \mathrm{g} / \mathrm{kg} / \mathrm{min})^{6}$ and ketamine $(10 \mu \mathrm{g} / \mathrm{kg} / \mathrm{min}){ }^{7}$ Additionally, a preoperative epidural anesthesia with bupivacaine $(0.5 \mathrm{mg} / \mathrm{kg})^{8}$ and morphine $(0.1 \mathrm{mg} / \mathrm{kg})^{9}$ and a intraoperative sciatic nerve block with lidocaine $(1 \mathrm{mg} / \mathrm{kg}){ }^{10}$ were performed. For postoperative analgesia the aforementioned CRI of fentanyl, lidocaine and ketamine was used for $24 \mathrm{~h}$, and carprofen $(4 \mathrm{mg} / \mathrm{kg})^{11}$ was initiated the day of the surgery and continued for 10 additional days.

The surgical procedure was performed by disarticulation of the hip, as described elsewhere [2]. The dogs remained in the hospital for approximately 5 days.

\section{Kinetic and kinematic gait evaluation}

Kinetic (forces) and kinematic (movement) gait analysis was performed one to three days before the amputation, as well as 10, 30, 90 and 120 days after surgery.

Kinetics were measured using a specially designed treadmill $^{12}$ consisting of four separate belts, each of them with an integrated force plate underneath. This design allowed the simultaneous measurement of all limb forces. 
Kinematic analysis was performed with the aid of retro-reflective markers $(\varnothing 16 \mathrm{~mm} \text { reflective markers })^{13}$ positioned on 24 anatomic landmarks (8 per remaining limb), using double-sided adhesive tape; the location of these markers has been previously described $[10,11]$ and is illustrated in Fig. 1. Six high-speed infrared cameras $^{14}$ were used to record marker movement in all three remaining limbs simultaneously, as the animals were walking at a controlled speed (measurement frequency: $100 \mathrm{~Hz}$ ). Before each measurement, static and dynamic camera calibration was performed using an L-shaped calibration device. $^{15}$
On each gait analysis session, patients were gently introduced to the gait on the treadmill; on the first day, a speed at which each individual patient walked comfortably on the treadmill was determined; on each subsequent session the patient was evaluated using the same speed, ranging from 0.5 to $0.8 \mathrm{~m} / \mathrm{s}$. During each gait analysis session, two to six trials were recorded, each with a duration of approximately $30 \mathrm{~s}$, until at least one valid trial was obtained. A valid trial was defined as 10 consecutive regular steps, in which the dog walked smoothly, without any external forces from the handler being applied, with all paws landing on the appropriate
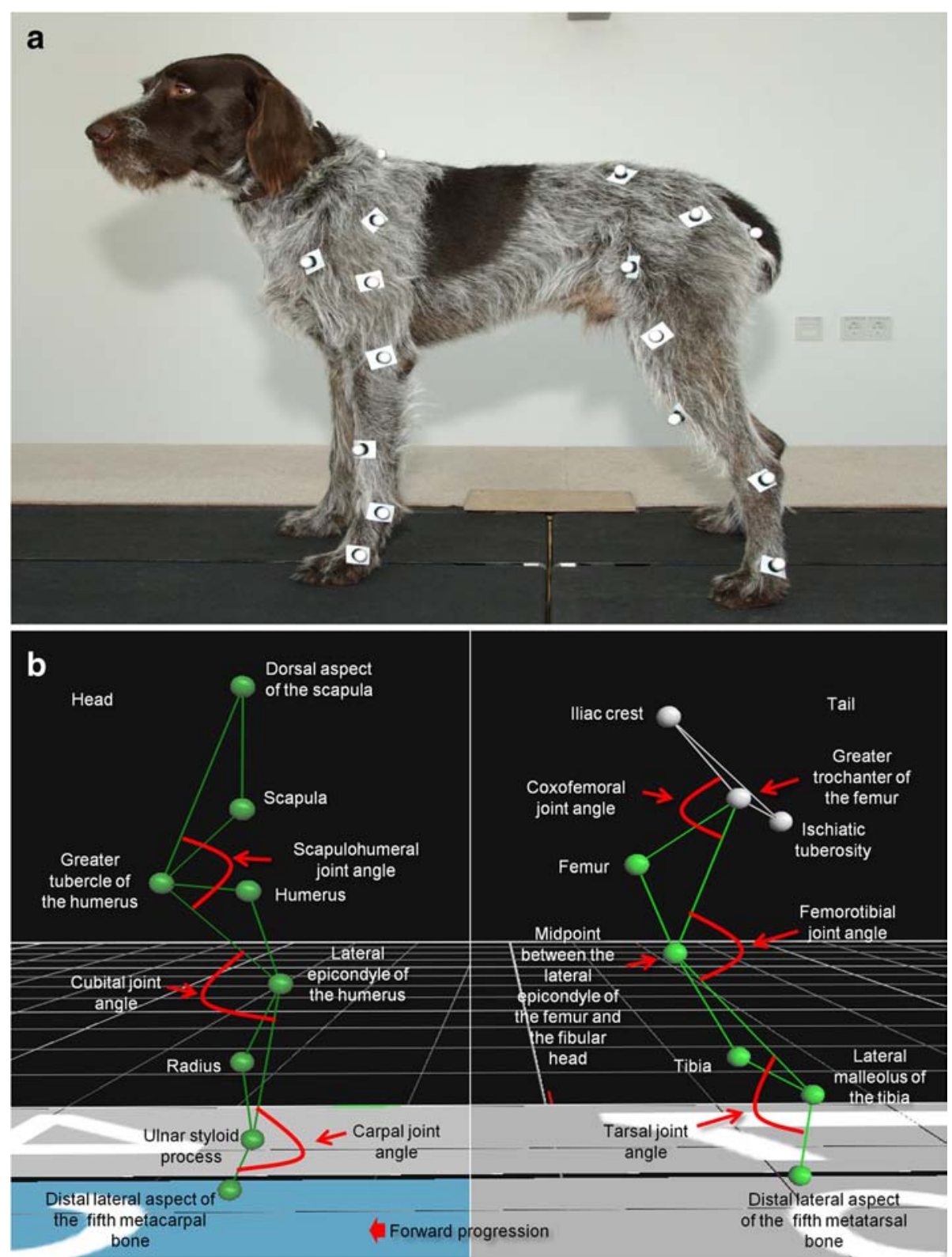

Fig. 1 a Example of the localization of the retro-reflective markers on a healthy patient; $\mathbf{b}$ Illustration of the localization of the retro-reflective markers on the anatomical reference points and the measured angles 
force plate, without overstepping. Video recording was performed, to ensure that the steps were appropriate for analysis.

Both kinetic and kinematic data were simultaneously recorded using commercially available software. ${ }^{16}$

Ten consecutive steps were afterwards analyzed for the following kinetic parameters: peak vertical force $(\mathrm{PFz})$, mean vertical force $(\mathrm{MFz})$, and vertical impulse (integral $[\mathrm{IFz}]$ ). All forces were normalized to the individual body weight of each dog and data were expressed as percentage of body weight (\%BW). Mean \pm standard deviation (SD) was calculated from 10 valid consecutive steps. Afterwards, load redistribution (LR) was calculated for each forelimb and remaining hind limb, for each measured parameter (PFz, MFz, IFz), using the following equation (according to Steiss et al. [12]): \% load bearing = $\mathrm{Fz}$ of the limb/total $\mathrm{Fz}$ of all limbs*100. Other kinetic parameters were not calculated to avoid overloading this section with too much information. The kinetic data were processed using commercial software ${ }^{17}$ and exported to a commercially available spreadsheet. ${ }^{18}$

In order to process the kinematic data in Vicon Nexus, all markers were labeled in a trial. Then, 10 valid foot strikes were marked manually to define the gait cycle (stance and swing phases) of each limb. Using a 2dimensional (2-D) model, projected flexion and extension angles of each remaining joint were calculated: contralateral (with respect to the amputated hind limb) scapulohumeral joint, contralateral cubital joint, contralateral carpal joint, ipsilateral (with respect to the amputated hind limb) scapulohumeral joint, ipsilateral cubital joint, ipsilateral carpal joint, contralateral coxofemoral joint, contralateral femorotibial joint and contralateral tarsal joint. Measured angles are illustrated in Fig. 1. In order to compare the movement pattern of each analyzed joint, the gait cycles were normalized to 100 in all dogs and displayed as percentage of one whole stride. The mean joint angle $( \pm \mathrm{SD})$ and the range of motion $( \pm \mathrm{SD})$ of the aforementioned joints were calculated from the mean joint angle progression curves (MJAPC) calculated from the 10 strides per dog. Mean joint angles and range of motion were used since the reader can easily understand their comparison between sessions and to avoid overloading the manuscript with too much data. The kinematic data were processed using commercial software ${ }^{19}$ and then exported to a commercially available spreadsheet ${ }^{18}$.

\section{MR evaluation of the contralateral stifle joint}

The MR examination was performed under general anesthesia before and 120 days after amputation. The anesthetic protocol was the same described above, excluding local anesthetics and CRIs. The animals were positioned in lateral recumbency with the limb to be examined in a non-dependent position and the stifle joint at an angle of $\sim 135^{\circ}$. Using a state-of-the-art $3 \mathrm{~T}$ MR scan,${ }^{20}$ images were obtained from the contralateral stifle. Small $(11 \mathrm{~cm} \varnothing)$ surface ring coils (Achieva $3.0 \mathrm{~T}$ Musculoskeletal SENSE Flex S coil 2 elements) were used as image enhancers; these were positioned parallel to each other, lateral and medial to the examined stifle, with the joint centered between the two coils. The MR protocol used included a 3-D (3-dimensional) PDW (proton-density weighted) acquisition sequence, which was afterwards reconstructed in sagittal, dorsal (parallel to patella ligament) and transversal (parallel to tibial plateau) planes (slices every $2 \mathrm{~mm}$ ), a PDW HR (highresolution) TSE (turbo spin echo) SENSE (sensitivity encoding for fast MR) sequence in sagittal plane (slices every $2 \mathrm{~mm}$ ), a PDW HR SPAIR (spectrally adiabatic inversion recovery) SENSE in sagittal plane (slices every $2 \mathrm{~mm}$ ), and a T1-weighted TSE clear (constant level appearance) sequence in sagittal plane (slices every $1.8 \mathrm{~mm}$ ).

This protocol had been previously standardized and regarded as suitable for use in clinical cases, since diagnostic image quality is optimal and acquisition time is only $22 \mathrm{~min}$ (total examination time is about $40 \mathrm{~min}$ including positioning, reference scan, survey, and sequence planning).

Using a high-resolution diagnostic screen ${ }^{21}$ the images were assessed by a trained evaluator (VGZ), who looked for changes in the signal intensity of the cranial cruciate ligament $(\mathrm{CrCL})$, the caudal cruciate ligament $(\mathrm{CdCL})$ and the lateral and medial menisci. Possible changes in the cartilage surfaces, as well as evidence of osteoarthritic changes were also evaluated in the lateral and medial femoral condyles, femoral trochlear groove, patella and tibial plateau.

It was expected that, due to a possible underlying metastatic disease, some patients could die or be euthanized before the end of the study; if that was the case, it was planned to ask the owner to authorize the MR examination postmortem.

\section{Owner evaluation of patient comfort}

The owner was requested to fill out an evaluation form (modified from Hielm-Björkman et al. [13]) before the amputation and 10, 30, 90 and 120 days after the procedure, in order to gather his/her (subjective) impressions with regard to patient comfort and recovery. At the end of the study (day 120), owners filled out a questionnaire to assess their final impression regarding the degree of activity and life quality of the dog, and their general impression of and satisfaction with the procedure; besides, owners were encouraged to make further comments. It was planned that if the animal died before the end of the study, an appropriate moment would be looked for to ask the owner to fill out the questionnaire. 
The questions of the questionnaire were adapted from Carberry and Harvey [4], Withrow and Hirsch [5], von Werthern et al. [6] and Kirpensteijn et al. [7]. The owners' assessment of patient comfort and the final questionnaire were made in German and translated into English as accurately as possible.

\section{Statistical methods}

Due to the small sample size and very heterogeneous patient population included in this study, it was decided to use non-parametric statistics. Thus, data were analyzed using a Kruskal-Wallis one-way ANOVA test to compare between sessions; when statistically significant differences were found, a Wilcoxon signed-rank test for paired observations was performed to determine which session was different. All tests were considered statistically significant if $p<0.05$ and were performed using standard statistical software. ${ }^{22}$ Descriptive statistics were calculated using a commercially available spreadsheet ${ }^{18}$, where appropriate.

\section{Results}

\section{Clinical data}

Breed, sex, age, reason to amputate and performed evaluations of the 12 patients enrolled in this study are illustrated in Table 1. As can be seen in this table, the most common reason for performing the amputation was a tumor, followed by trauma and one surgical complication. Six right and six left hind limbs were amputated. Nine patients survived until the end of the study. Due to the underlying metastatic disease, one animal (patient 5) was euthanized 36 days after the amputation and another one (patient 6) died 120 days after the procedure. One dog (patient 8) died unexpectedly 22 days after the amputation due to abdominal bleeding caused by a previously asymptomatic and undiagnosed hepatic hemangiosarcoma.

Patient 12 presented bilateral hip osteoarthritis; however, it was asymptomatic, and no signs of pain, lameness or difficulty to stand up were detected before or after the amputation. All other patients showed no abnormalities in the physical examination of the remaining limbs. No patient showed abnormalities on the physical examination of the spine throughout the study.

\section{Kinetic and kinematic gait evaluation}

The results of the kinetic and kinematic evaluations are presented in Figs. 2, 3, 4 and 5 and Tables 3 and 4. It should be noted that, although nine patients survived until the last examination day, the kinetic and kinematic data were not available from all of them. One animal (Patient 7) refused to walk on the treadmill and others (e.g. Patient 4) walked intermittently in such a way that some trials were not valid for analysis; even though these animals could walk and run perfectly fine on solid ground, they were afraid of walking on the treadmill, apparently due to the movement of the belts. Moreover, although all owners were extremely cooperative, when some of them had the impression that their dog was afraid or tired, they were reluctant to allow their pets to be walked on the treadmill long enough to record valid trials. The number of patients evaluated on each session is also indicated in Tables 3 and 4 .

Kinetic data showed that 10 days after amputation there was redistribution of the load to all remaining limbs (Fig. 2 and Table 2). The values and pattern of load shifting are represented in Fig. 2. The recorded PFz, MFz and IFz values showed no remarkable changes during the remaining examination time points, indicating that 10 days after the amputation the patient had already reached its

Table 1 Patients included in this study

\begin{tabular}{|c|c|c|c|c|c|c|c|c|c|c|c|}
\hline \multirow[t]{2}{*}{ Patient } & \multirow[t]{2}{*}{ Breed } & \multirow[t]{2}{*}{ Sex } & \multirow{2}{*}{$\begin{array}{l}\text { Age } \\
\text { (Years) }\end{array}$} & \multirow{2}{*}{$\begin{array}{l}\text { Weight } \\
(\mathrm{kg})\end{array}$} & \multirow[t]{2}{*}{ Reason to amputate } & \multicolumn{5}{|c|}{ Gait analyses } & \multirow[t]{2}{*}{ PO MR } \\
\hline & & & & & & Pre & 10 & 30 & 90 & 120 & \\
\hline 1 & Boxer & Male & 8 & 32 & Osteosarcoma & + & + & + & + & + & + \\
\hline 2 & Labrador & Female & 3 & 31 & Rhabdomyosarcoma & + & + & + & + & + & + \\
\hline 3 & Mixed-breed dog & Female & 4 & 32 & Osteosarcoma & + & + & + & + & + & + \\
\hline 4 & Mixed-breed dog & Male & 1 & 20 & Severe soft tissue trauma & + & + & + & + & + & + \\
\hline 5 & Mixed-breed dog & Male & 12 & 31 & Osteosarcoma & + & + & + & $E$ & - & - \\
\hline 6 & Swiss Mountain dog & Female & 10 & 39 & Osteosarcoma & + & + & + & + & $E$ & - \\
\hline 7 & Bernese Mountain dog & Male & 2 & 40 & Femoral fracture nonunion & - & - & - & - & - & + \\
\hline 8 & German Shepherd mix & Male & 7 & 26 & Severe soft tissue trauma & + & + & $E$ & - & - & - \\
\hline 9 & Mixed-breed dog & Female & 8 & 13 & Osteosarcoma & + & + & + & + & + & + \\
\hline 10 & Mixed-breed dog & Female & 11 & 8 & Malignant sarcoma & + & + & + & + & + & + \\
\hline 11 & Landseer & Female & 2 & 54 & Fibrosarcoma & + & + & + & + & + & - \\
\hline 12 & Mixed-breed dog & Female & 8 & 49 & Osteosarcoma & + & + & + & + & + & + \\
\hline
\end{tabular}

PO MR: Postoperative magnetic resonance scan; +: Performed; -: Not performed; E: Euthanasia 


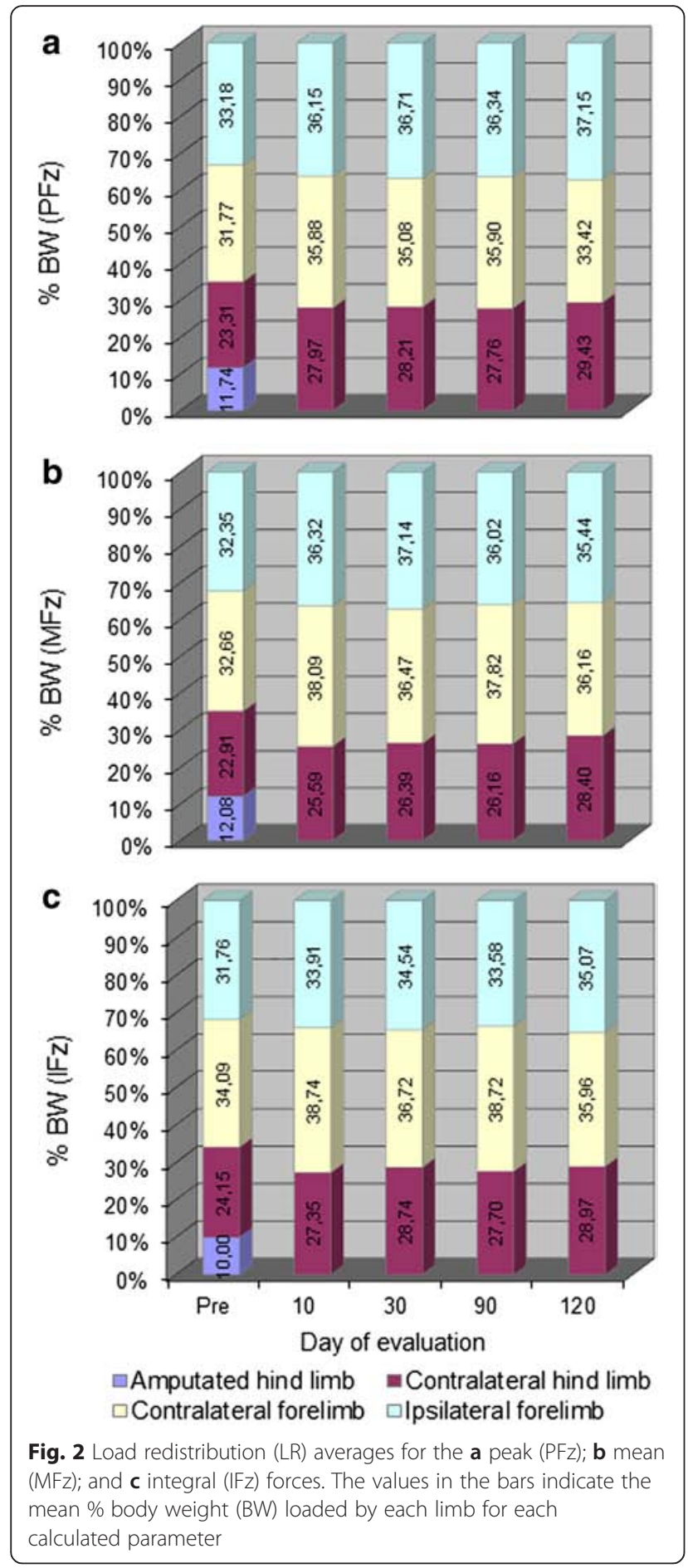

new locomotory pattern. This was true for all patients including the lightest $(8 \mathrm{~kg})$ and the heaviest $(54 \mathrm{~kg})$ ones. There were no statistically-significant differences between sessions (Table 2).

With regard to the kinematic gait analysis, even though the patients walked smoothly on the treadmill (Additional file 1: Video 1), there were significant differences between
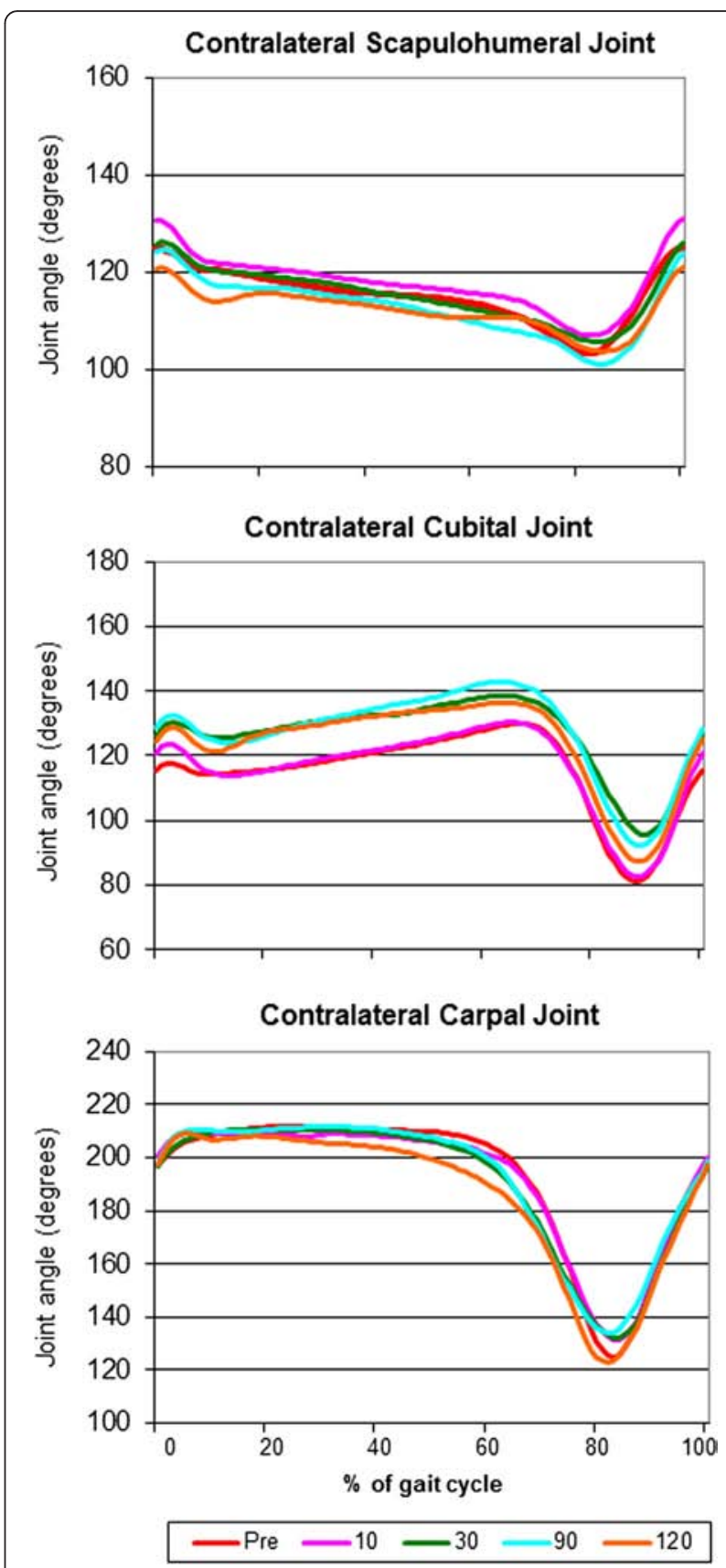

Fig. 3 Mean joint angle progression curves of the contralateral (with respect to the amputated hind limb) scapulohumeral joint, cubital joint and carpal joint. Note the similarity of the curves before and after amputation

sessions in the means of almost all joint angles (Table 3). It is important to note that there were also marked variations within a patient in the same session (not shown in Table 3). The MJAPC showed a similar pattern between sessions (Figs. 3, 4 and 5), but these showed marked individual variations (not shown).

Despite all these different kinematic results, ROMs of all analyzed joints were very similar before and after 


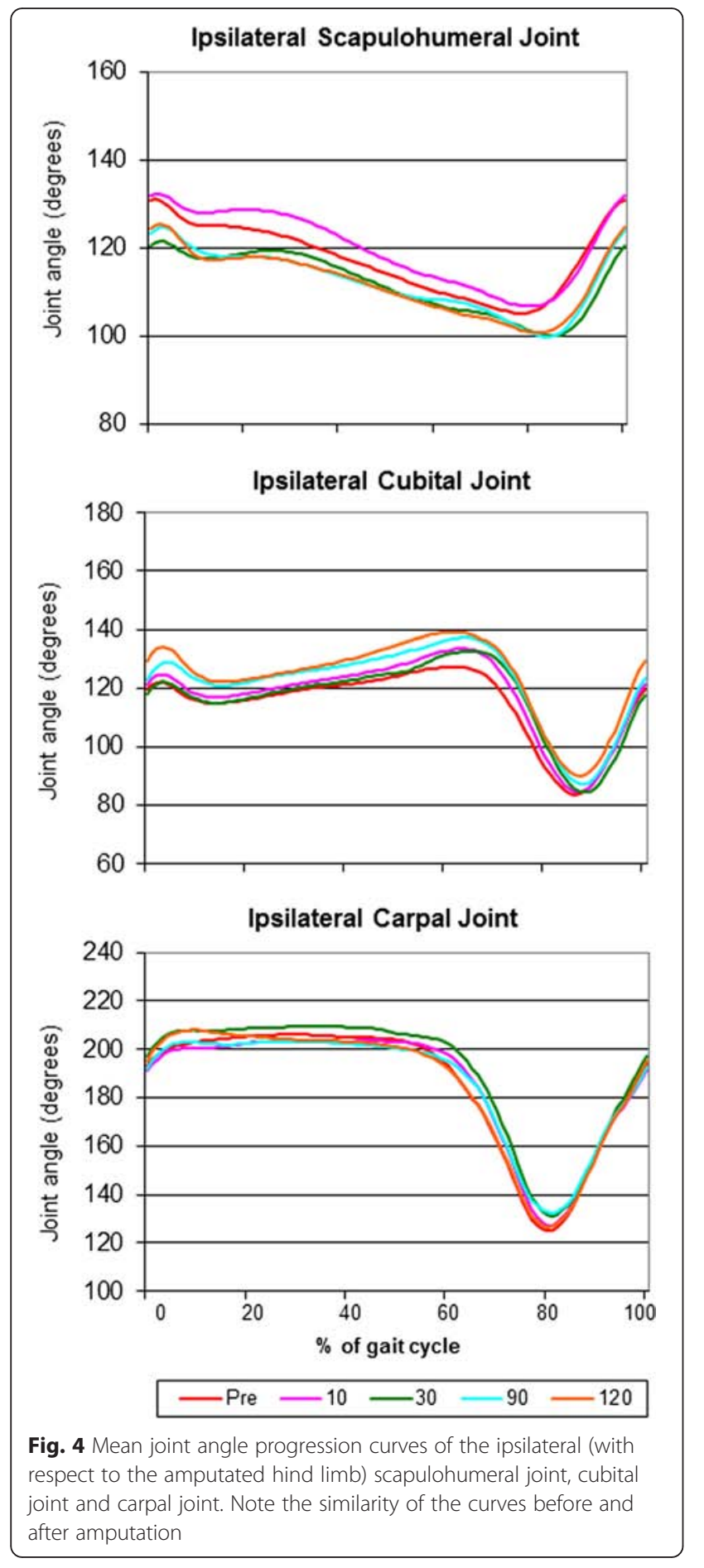

amputation and remained constant in the subsequent sessions after the amputation, without significant differences between sessions (Table 3).

\section{MR evaluation of the contralateral stifle joint}

Postoperative MR examination was possible in eight patients. Although nine patients survived until the end of the study, severe metastatic disease was detected in
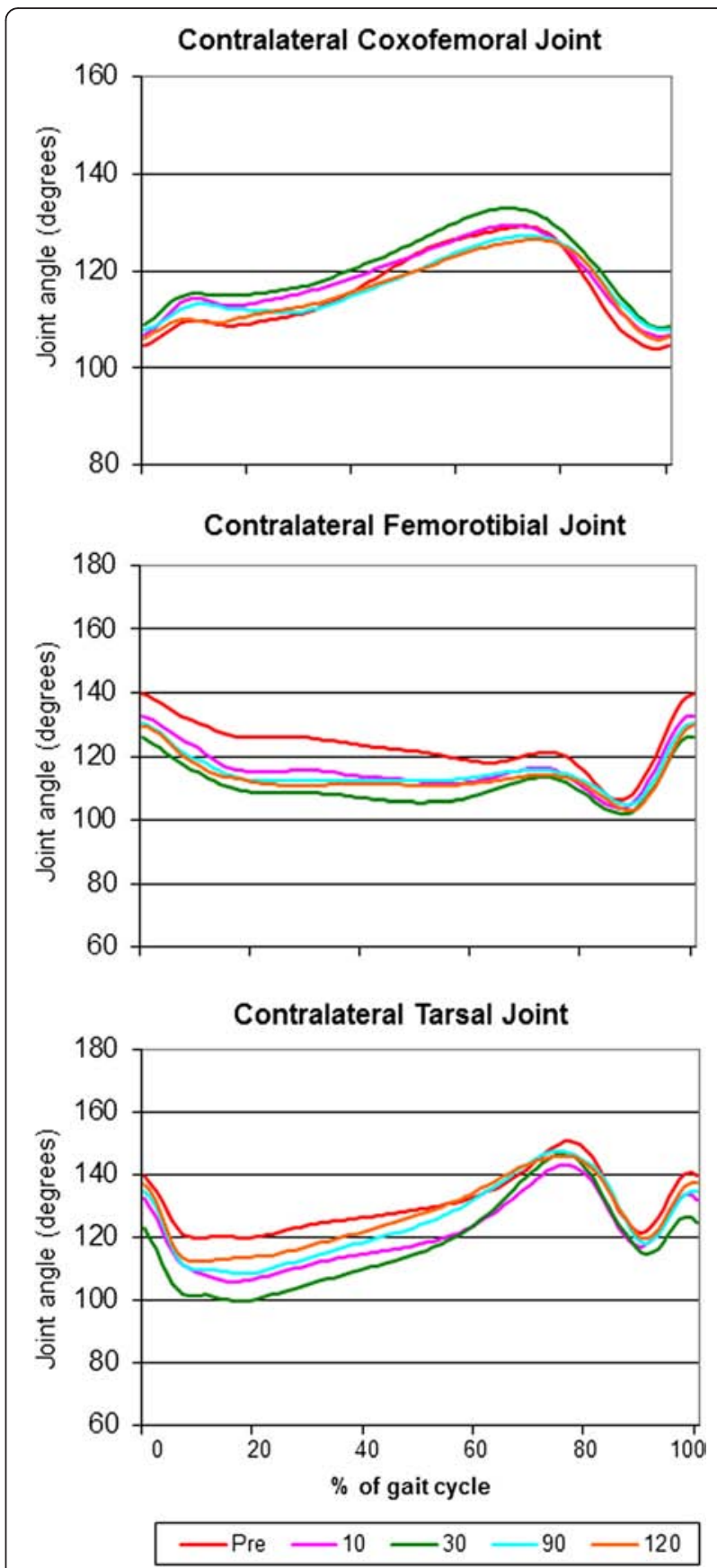

Fig. 5 Mean joint angle progression curves of the contralateral (with respect to the amputated hind limb) coxofemoral joint, femorotibial joint and tarsal joint. Note the similarity of the curves before and after amputation

patient 11 on day 120 , and the MR examination was not performed. Postmortem MR examination was not possible in any case, as the owners were very sensitive about their pet's death, and they elected to dispose the patients' dead bodies themselves. No changes in the signal intensity of the $\mathrm{CrCL}, \mathrm{CdCL}$ or the lateral and medial menisci were found, in comparison with the preoperative MR 
Table 2 Results of the kinetic analysis

\begin{tabular}{|c|c|c|c|c|c|c|c|}
\hline & & $\begin{array}{l}\text { Pre } \\
(n=11)\end{array}$ & $\begin{array}{l}10 \\
(n=11)\end{array}$ & $\begin{array}{l}30 \\
(n=10)\end{array}$ & $\begin{array}{l}90 \\
(n=9)\end{array}$ & $\begin{array}{l}120 \\
(n=8)\end{array}$ & $p$ \\
\hline \multicolumn{8}{|l|}{ PFz contralateral forelimb } \\
\hline & Mean & 64.88 & 71.45 & 67.69 & 69.78 & 66.74 & 0.252 \\
\hline & SD & 7.00 & 4.73 & 8.71 & 6.44 & 8.27 & \\
\hline \multicolumn{8}{|l|}{ PFz ipsilateral forelimb } \\
\hline & Mean & 67.78 & 71.97 & 70.86 & 70.64 & 74.18 & 0.796 \\
\hline & SD & 9.03 & 5.90 & 9.29 & 5.60 & 11.56 & \\
\hline \multicolumn{8}{|l|}{ PFz contralateral hind limb } \\
\hline & Mean & 47.60 & 55.68 & 54.44 & 53.96 & 58.75 & 0.454 \\
\hline & SD & 11.71 & 10.07 & 9.62 & 13.69 & 15.36 & \\
\hline \multicolumn{8}{|l|}{ MFz contralateral forelimb } \\
\hline & Mean & 47.55 & 52.71 & 49.33 & 50.70 & 48.49 & 0.327 \\
\hline & SD & 7.13 & 4.71 & 6.84 & 4.37 & 7.45 & \\
\hline \multicolumn{8}{|l|}{ MFz ipsilateral forelimb } \\
\hline & Mean & 47.10 & 50.27 & 50.25 & 48.30 & 47.53 & 0.816 \\
\hline & SD & 6.93 & 5.30 & 7.79 & 3.43 & 9.55 & \\
\hline \multicolumn{8}{|l|}{ MFz contralateral hind limb } \\
\hline & Mean & 33.37 & 35.41 & 35.70 & 35.08 & 38.09 & 0.865 \\
\hline & SD & 7.02 & 6.27 & 6.42 & 10.38 & 10.48 & \\
\hline \multicolumn{8}{|l|}{ IFz contralateral forelimb } \\
\hline & Mean & 30.28 & 32.09 & 30.41 & 30.07 & 28.82 & 0.945 \\
\hline & SD & 7.28 & 7.46 & 9.10 & 8.45 & 7.54 & \\
\hline \multicolumn{8}{|l|}{ IFz ipsilateral forelimb } \\
\hline & Mean & 28.20 & 28.09 & 28.61 & 26.08 & 28.10 & 0.967 \\
\hline & SD & 5.90 & 7.12 & 6.57 & 6.31 & 6.81 & \\
\hline \multicolumn{8}{|l|}{ IFz contralateral hind limb } \\
\hline & Mean & 21.45 & 22.65 & 23.81 & 21.51 & 23.21 & 0.999 \\
\hline & SD & 7.28 & 7.00 & 8.19 & 5.23 & 7.62 & \\
\hline
\end{tabular}

$p: p$ value of the Kruskal-Wallis test; PFz: Peak vertical force; MFz: Mean vertical force; IFz: vertical impulse (integral); SD: Standard deviation

images. No changes in the cartilage surface and no evidence of osteoarthritic changes were found (Fig. 6).

\section{Owner evaluation of patient comfort}

The results of the owners' assessment of patient comfort are presented in Table 4 and show a clear tendency of the patients to improve after amputation. Patient movement after amputation is exemplified with Additional file 2: Video 2. Ten owners answered the final questionnaire (Table 5). This questionnaire revealed a high degree of owner satisfaction with the amputation result.

\section{Discussion}

The main goal of this study was to provide owners and veterinarians with accurate, objective information, not existing previously, about the outcome of the amputated patient (i.e. how the dog adapted to the new locomotory status).

Examination times were chosen using the data of a previous study [7] which indicated that most dogs adapted to the amputation within a month, some within a week, and all within 3 months after surgery. With the last follow-up 120 days after the procedure, a final attempt was made to find any evidence of further gait changes. Since there is the belief that orthopedic disease might occur in the remaining limb after amputation, as a result of a theoretical overload, the contralateral stifle was morphologically assessed using MR images, to evaluate this likelihood. The femorotibial (stifle) joint was chosen, instead of the coxofemoral or tarsal joint, as the former has been extensively investigated, and there is a whole body of literature exemplifying normal and pathologic MR images to compare [14-19]. 
Table 3 Results of the kinematic analysis

\begin{tabular}{|c|c|c|c|c|c|c|c|}
\hline & & Pre $(n=11)$ & $10(n=11)$ & $30(n=10)$ & $90(n=9)$ & $120(n=8)$ & $p$ \\
\hline \multirow[t]{2}{*}{ Contralateral scapulohumeral joint } & Mean \pm SD & $115.2 \pm 5.67$ & $117.9 \pm 5.83$ & $115.4 \pm 5.54$ & $112.7 \pm 6.09$ & $112.3 \pm 4.24$ & $<0.001$ \\
\hline & $\mathrm{ROM} \pm \mathrm{SD}$ & $27.68 \pm 7.48$ & $31.46 \pm 8.12$ & $29.51 \pm 6.73$ & $31.49 \pm 6.26$ & $26.07 \pm 6.04$ & 0.478 \\
\hline \multirow[t]{2}{*}{ Contralateral cubital joint } & Mean \pm SD & $114.9 \pm 12.93$ & $115.9 \pm 12.82$ & $126.5 \pm 11.59$ & $127.2 \pm 13.67$ & $123.6 \pm 13.91$ & $<0.001$ \\
\hline & $\mathrm{ROM} \pm \mathrm{SD}$ & $57.98 \pm 15.56$ & $61.8 \pm 11.64$ & $56.51 \pm 14.74$ & $60.75 \pm 13.06$ & $62.74 \pm 9.60$ & 0.898 \\
\hline \multirow[t]{2}{*}{ Contralateral carpal joint } & Mean \pm SD & $192.2 \pm 26.85$ & $191.5 \pm 24.89$ & $190.1 \pm 26.03$ & $191.5 \pm 25.66$ & $185.8 \pm 26.88$ & $<0.001$ \\
\hline & $\mathrm{ROM} \pm \mathrm{SD}$ & $99.38 \pm 19.62$ & $97.45 \pm 11.28$ & $104.7 \pm 10.83$ & $94.25 \pm 13.65$ & $101.1 \pm 8.17$ & 0.666 \\
\hline \multirow[t]{2}{*}{ Ipsilateral scapulohumeral joint } & Mean \pm SD & $117.5 \pm 7.92$ & $120.1 \pm 8.33$ & $111.9 \pm 6.87$ & $112.4 \pm 6.94$ & $112.1 \pm 7.12$ & $<0.001$ \\
\hline & $\mathrm{ROM} \pm \mathrm{SD}$ & $34.11 \pm 5.69$ & $32.2 \pm 4.35$ & $29.77 \pm 4.80$ & $32.17 \pm 2.71$ & $31.06 \pm 5.03$ & 0.654 \\
\hline \multirow[t]{2}{*}{ Ipsilateral cubital joint } & Mean \pm SD & $114.6 \pm 12.28$ & $117.6 \pm 13.33$ & $116.7 \pm 12.94$ & $121.5 \pm 13.63$ & $123.8 \pm 13.58$ & $<0.001$ \\
\hline & $\mathrm{ROM} \pm \mathrm{SD}$ & $55 \pm 11.81$ & $57.96 \pm 11.92$ & $57.23 \pm 12.69$ & $59.84 \pm 12.73$ & $57.78 \pm 11.82$ & 0.916 \\
\hline \multirow[t]{2}{*}{ Ipsilateral carpal joint } & Mean \pm SD & $185.5 \pm 26.36$ & $185.6 \pm 24.54$ & $190.6 \pm 25.54$ & $185.9 \pm 23.23$ & $185.5 \pm 26.05$ & $<0.001$ \\
\hline & $\mathrm{ROM} \pm \mathrm{SD}$ & $92.65 \pm 19.14$ & $91.83 \pm 18.76$ & $91.01 \pm 15.71$ & $82.42 \pm 12.51$ & $93.36 \pm 10.05$ & 0.630 \\
\hline \multirow[t]{2}{*}{ Contralateral coxofemoral joint } & Mean \pm SD & $116.1 \pm 8.37$ & $118.4 \pm 6.96$ & $120.8 \pm 7.516$ & $116.8 \pm 6.29$ & $116.1 \pm 6.64$ & $<0.001$ \\
\hline & $\mathrm{ROM} \pm \mathrm{SD}$ & $30.25 \pm 6.22$ & $27.47 \pm 9.90$ & $30.08 \pm 9.71$ & $25.17 \pm 9.09$ & $24.89 \pm 6.27$ & 0.501 \\
\hline \multirow[t]{2}{*}{ Contralateral femorotibial joint } & Mean \pm SD & $123.2 \pm 7.48$ & $115.8 \pm 6.62$ & $110.4 \pm 5.76$ & $114.7 \pm 5.55$ & $113.3 \pm 5.68$ & $<0.001$ \\
\hline & $\mathrm{ROM} \pm \mathrm{SD}$ & $42.4 \pm 4.28$ & $37.67 \pm 8.44$ & $37.82 \pm 7.34$ & $40.8 \pm 12.16$ & $34.83 \pm 8.31$ & 0.299 \\
\hline \multirow[t]{2}{*}{ Contralateral tarsal joint } & Mean \pm SD & $130.5 \pm 8.98$ & $121 \pm 10.93$ & $117.8 \pm 14.07$ & $124.9 \pm 12.21$ & $127.2 \pm 10.75$ & $<0.001$ \\
\hline & $\mathrm{ROM} \pm \mathrm{SD}$ & $47.74 \pm 10.2$ & $50.57 \pm 10.52$ & $61.27 \pm 14.25$ & $57.73 \pm 11.24$ & $48.04 \pm 14.17$ & 0.143 \\
\hline
\end{tabular}

Mean: mean joint angle calculated from the mean joint angle progression curves; SD: Standard deviation; ROM $=$ Range of Motion; $p=p$ value of the Kruskal-Wallis test

As expected, a very heterogeneous population was found in this study: different breeds, ages and weights. The main reason for amputating a patient was the presence of a tumor, which agrees with previous studies [6, 7]. The mortality seen in this study was not related the amputation itself.
The kinetic and kinematic analyses revealed that the patients had begun adapting to the new locomotory situation even before the amputation was performed, and that 10 days after the procedure, all major changes had already taken place. It was unfortunate that it was not possible to perform all examinations on all patients.
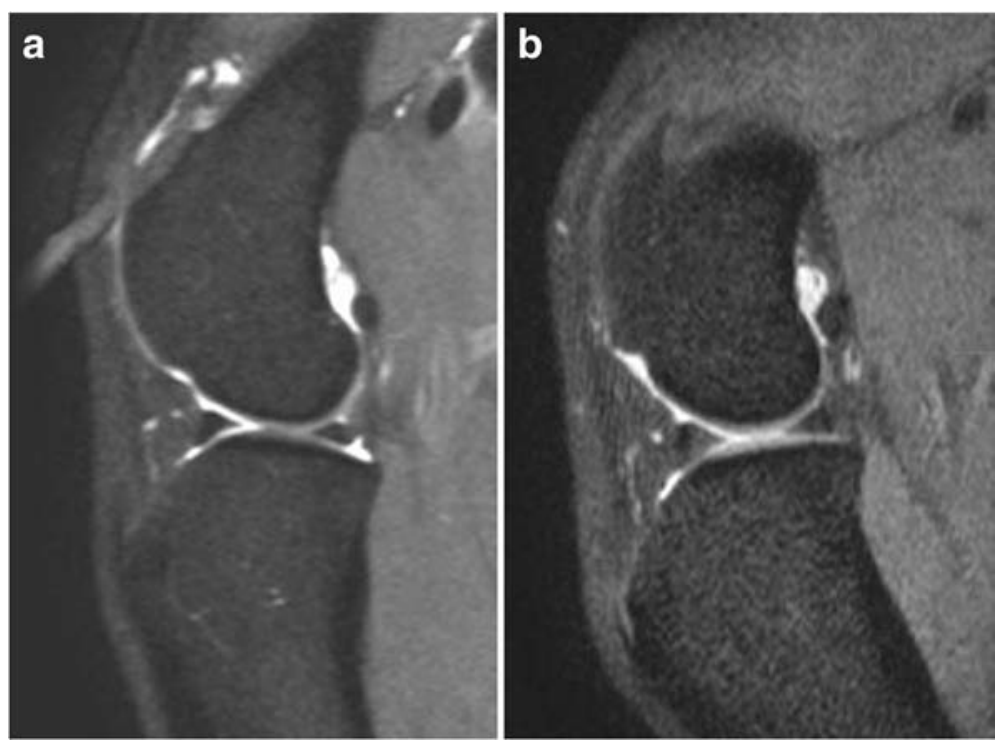

Fig. 6 Example of the magnetic resonance examination of the stifle in one patient before (a) and 120 days after the amputation (b). No changes could be detected in the joint 120 days after the procedure 
Table 4 Owner assessment of patient comfort $^{a}$ (first part)

\begin{tabular}{|c|c|c|c|c|c|}
\hline & Pre $(n=12)$ & $10(n=12)$ & $30(n=11)$ & $90(n=10)$ & $120(n=10)$ \\
\hline \multicolumn{6}{|l|}{ Attitude } \\
\hline Very bright (score 0) & 4 & 2 & 6 & 4 & 3 \\
\hline Alert (score 1) & 5 & 8 & 5 & 6 & 7 \\
\hline Neither alert nor indifferent (score 2) & 3 & 2 & 0 & 0 & 0 \\
\hline Indifferent (score 3) & 0 & 0 & 0 & 0 & 0 \\
\hline Depressed (score 4) & 0 & 0 & 0 & 0 & 0 \\
\hline Cumulative score for attitude & 11 & 12 & 5 & 6 & 7 \\
\hline \multicolumn{6}{|l|}{ Willingness to move (general) } \\
\hline Very willing (score 0) & 2 & 3 & 6 & 5 & 5 \\
\hline Willing (score 1) & 4 & 8 & 5 & 4 & 5 \\
\hline Hesitant (score 2) & 4 & 1 & 0 & 1 & 0 \\
\hline Reluctant (score 3) & 1 & 0 & 0 & 0 & 0 \\
\hline Does not move (score 4) & 1 & 0 & 0 & 0 & 0 \\
\hline Cumulative score for willingness to move (general) & 19 & 10 & 5 & 6 & 5 \\
\hline \multicolumn{6}{|l|}{ The $\operatorname{dog} \ldots$} \\
\hline \multicolumn{6}{|l|}{.. lies down. } \\
\hline Easily (score 0) & 3 & 4 & 7 & 9 & 9 \\
\hline Carefully (score 1) & 5 & 7 & 4 & 1 & 1 \\
\hline Slowly (score 2) & 1 & 1 & 0 & 0 & 0 \\
\hline with difficulty (score 3) & 2 & 0 & 0 & 0 & 0 \\
\hline with a lot of difficulty (score 4) & 1 & 0 & 0 & 0 & 0 \\
\hline Cumulative score for the dog lies down... & 17 & 7 & 4 & 1 & 1 \\
\hline \multicolumn{6}{|l|}{$\ldots$ stands up ... } \\
\hline Easily (score 0 ) & 4 & 2 & 4 & 5 & 6 \\
\hline Carefully (score 1) & 3 & 10 & 6 & 4 & 2 \\
\hline Slowly (score 2) & 2 & 0 & 1 & 1 & 2 \\
\hline with difficulty (score 3) & 2 & 0 & 0 & 0 & 0 \\
\hline with a lot of difficulty (score 4) & 1 & 0 & 0 & 0 & 0 \\
\hline Cumulative score for the dog stands up... & 17 & 10 & 8 & 6 & 6 \\
\hline \multicolumn{6}{|l|}{ Willingness to move after resting } \\
\hline Very willing (score 0) & 0 & 4 & 4 & 4 & 4 \\
\hline Willing (score 1) & 5 & 7 & 6 & 5 & 5 \\
\hline Hesitant (score 2) & 6 & 0 & 1 & 1 & 1 \\
\hline Reluctant (score 3) & 1 & 1 & 0 & 0 & 0 \\
\hline Does not move (score 4) & 0 & 0 & 0 & 0 & 0 \\
\hline Cumulative Score for willingness to move after resting & 20 & 10 & 8 & 7 & 7 \\
\hline \multicolumn{6}{|l|}{ Willingness to move after exercising } \\
\hline Very willing (score 0) & 0 & 0 & 1 & 3 & 2 \\
\hline Willing (score 1) & 3 & 9 & 6 & 4 & 6 \\
\hline Hesitant (score 2) & 4 & 2 & 3 & 3 & 2 \\
\hline Reluctant (score 3) & 3 & 1 & 1 & 0 & 0 \\
\hline Does not move (score 4) & 2 & 0 & 0 & 0 & 0 \\
\hline Cumulative score for willingness to move after exercising & 28 & 16 & 15 & 10 & 10 \\
\hline Mean score (sum of cumulative scores / patients evaluated) & 9.3 & 5.4 & 4.1 & 3.6 & 3.6 \\
\hline
\end{tabular}

${ }^{\mathrm{a}}$ Modified from Hielm-Björkman et al. [13] 
Table 5 Final owner questionnaire $(n=10)$

\begin{tabular}{|c|c|c|c|}
\hline \multicolumn{4}{|c|}{ 1. Please indicate how satisfied you are with the results of the amputation } \\
\hline Very satisfied: 8 & Satisfied: 2 & Not satisfied: 0 & Very dissatisfied: 0 \\
\hline \multicolumn{4}{|c|}{ 2. Please indicate how well the dog adapted to the amputation } \\
\hline Very good: 7 & Good: 2 & Fair: 1 & Poor: 0 \\
\hline \multicolumn{4}{|l|}{ 3. The time the dog took to recover was: } \\
\hline More than expected: 0 & As expected: 3 & & Less than expected: 7 \\
\hline \multicolumn{4}{|l|}{ 4. Were behavioral changes observed? } \\
\hline Yes: 3 & & & No: 7 \\
\hline \multicolumn{4}{|c|}{ 5. Would you make a similar decision in the future, in case an amputation would be needed in another pet? } \\
\hline Yes: 10 & Not sure: 0 & & No: 0 \\
\hline \multicolumn{4}{|c|}{ 6. Before the amputation, were you reluctant to allow this procedure being performed in your dog? } \\
\hline Yes: 8 & & & No: 2 \\
\hline \multicolumn{4}{|c|}{ 7. The degree of activity of your dog after the amputation was: } \\
\hline Increased: 2 & The same: 6 & & Decreased: 2 \\
\hline \multicolumn{4}{|c|}{ 8. In general, you think that the life quality of the dog after the amputation: } \\
\hline Increased: 6 & Is the same: 4 & & Decreased: 0 \\
\hline
\end{tabular}

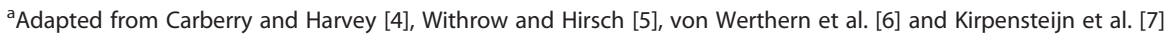

However, exclusively using "good" trials allowed us to obtain reliable results. It should be noted that, even though a valid trial implied that the handler did not exert any external forces, it was not possible to have the animals completely free on the treadmill (see Additional file 1: Video 1). Unfortunately, the willingness to walk or trot or overall activity of the patient were not evaluated in the present study; these factors would have brought additional information about the outcome. It is remarkable that the largest patient (patient 11-54 kg) and the smallest one (patient $10-8 \mathrm{~kg}$ ) adapted equally well. These results agree with the study of Kirpensteijn et al. [7], indicating that, even though subjective, the observations made by the owners of such study were actually very precise. The present study has the advantage of looking at the patients objectively and prospectively, leaving no doubt about the fast adaptation of all animals. The fact that there were no significant changes in the load redistribution after amputation was initially surprising; however, it is easily explainable: all patients were severely lame before the amputation, meaning that the adaptation and compensation to the lack of a hind limb had begun to take place before the amputation was performed. This was even clearer when looking only at those patients which did not load the affected limb at all before surgery. It should also be kept in mind, that the lack of statistically significant changes could have resulted from the low number of dogs included in this study, and therefore in the lack of power of the tests.

The kinematic results (including the statistical analysis) should be interpreted with caution. The fact that significant differences were found in the absolute values of the different joint angles could be explained by the normal variation inherent to motion analysis: marker localization changes lead to changes in joint angles measured. Even though every effort was made to place the 
markers in the right position, small variations might have occurred, leading to different measurements. However, also huge variations were seen within a session (not shown in Table 3 or Figs. 3, 4 and 5), possibly indicating that the patients adapted to every step they made and in a very irregular manner. Affected balance of the dog during movement and the resulting instability might cause these variations. Another possible explanation is the fact that several gait patterns are possible at a given speed and by a permanent adjustment of the speed of the animal to the treadmill speed [20]. It should also be taken into account that slow speeds were used before the amputation, in order to avoid worsening the pain that patients were already experiencing. After the amputation the animals could have walked on the treadmill more comfortably at faster speeds (personal observations). Even though this might have caused an irregular walking pattern after the procedure, speeds were kept constant to avoid adding a variance factor when measuring the GRF [21]. Finally, the angles measured here can be used to illustrate the movement patterns for the patients in this study, and they cannot be extrapolated to other patient populations. However, our study focuses on determining whether there are variations in the different kinematic parameters before and after amputation in this particular patient population, and that does not seem to be the case.

The similarity in the ROMs before and after surgery is a remarkable finding. The measurement of ROMs seems to be less susceptible to the sources of error commonly found in kinematic studies (misplacement of markers and skin movement), at least in the hind limb [22]. Therefore, the results of the ROMs are more accurate than the absolute measurement of joint angles. That being said, the lack of significant differences between sessions suggests that the patient had also begun to adapt to the new movement situation before amputation, and that this remained stable after the procedure. However, it should also be taken into consideration that joint angular curves in each measurement points are very similar in shape, resulting in stable ROMs; this could also explain why the ROMs showed much less variation than mean joint angles.

The lack of changes in the MR examination of the remaining contralateral stifle after amputation could indicate that an overload in the remaining contralateral hind limb, leading to joint pathology, is not very likely. It was decided to investigate this point, as it is commonly believed by the general public, and even by some veterinarians, that amputating a dog might predispose it to orthopedic abnormalities; the results of our MR (and also our physical examinations) proved otherwise, at least for the stifle. Although 4 months after amputation might possibly be a short time to evaluate joint changes, a previous study describing the experimentally induced rupture of the $\mathrm{CrCL}$ in 5 crossbred dogs showed that it is possible to see changes in the cartilage and subchondral bone as early as 4 weeks after the rupture [17]. It is of course difficult to extrapolate such findings to this study, but it could indicate that, if there were ongoing changes in joint morphology, they would be visible 4 months after amputation. Besides, most of our research subjects were oncologic patients, with a (likely) short life span. Measuring cartilage thickness would have been a more accurate method to evaluate subtle joint changes [17]. Unfortunately, this could not be done due to software limitations. In any case, we did not expect to see any changes, as our clinical experience indicates that no changes are seen in the contralateral limb after a hindlimb amputation; nevertheless, it is not known what kind of changes an amputation would cause in patients suffering from degenerative joint disease in the remaining limbs.

With regard to the owner evaluation assessment of the patient after the procedure, the results were as expected: in Table 4, the positive outcome of most patients can be clearly seen. The improvement is especially remarkable in the ability of the dog to lie down and stand up. The lack of improvement or worsening of some parameters for some patients seemed to be related more to the declining general condition of the patient, than to the effects of the amputation itself. The use of an objective tool to measure patient activity, such as an accelerometer, could have provided more accurate information about the outcome and they have been previously used in animals [23]; however, this tool was not available to us.

The responses to the final questionnaire were also as expected: most owners were initially reluctant to have their dogs amputated, but were satisfied with the overall result and the quality of life was considered good; this is in agreement with other studies [4-7]. In the present study, some owners even considered that their pet's quality of life improved after the amputation, and this might have been related to the removal of the source of pain. The behavioral changes reported by the owners in this study were more small disabilities than behavioral problems. The behavioral problems previously reported [7], such as aggression and anxiety, were not seen in the patients of the present study.

As in previous studies [4, 7], all owners responded that they would have another pet amputated, and none regretted the decision. We believe that an evaluation made by owners whose pets died shortly after the procedure would have been negatively biased and they were not gathered.

The favorable responses of most owners can be explained by the fact that the dogs adapted very soon to their new locomotory situation (kinetic and kinematic 
analyses) and to the lack of morphologic changes in other joints (as it might be inferred from the lack of morphologic changes in the stifle).

\section{Limitations}

Our study provides new information; however, there were important limitations in this study: the lack of a homogeneous population prevented us from comparing the kinetic and kinematic data with other studies looking at normal patient populations or using a control group. However, it is virtually impossible to perform such a clinical study using a homogeneous population. Additionally, kinematic data are breed-specific [24], and not all breeds have been studied yet. In any case, in the present study there were a high number of mixed-breed dogs, which are very difficult to characterize. The small sample size is another important limitation, which also possibly led to lack of statistical power. The effect of missing measurements for some patients might have also obscured the results. Finally, even though the evaluator was experienced in reading MR images of canine stifles, the lack of a board-certified radiologist for interpretation of the images may have also been a limitation of this study.

\section{Conclusions}

In spite of the limitations, this study provides objective evidence indicating that dogs have a quick adaptation process after a hind limb amputation. The adaptative processes to the new locomotion begin even before the amputation is performed. Since the veterinarian is responsible for providing accurate information before an amputation [2], we strongly believe that this study provides useful information, that will allow veterinarians the possibility to give dog owners more realistic expectations of a hind limb amputation.

\section{Endnotes}

${ }^{1}$ L-Polamivet ${ }^{\oplus}$ : Intervet Deutschland GmbH, Unterschleißheim, Germany

${ }^{2}$ Diazepam-ratiopharm": Ratiopharm GmbH, Ulm, Germany

${ }^{3} \mathrm{Narcofol}^{\circledR} 10 \mathrm{mg} / \mathrm{mL}$ : CP-Pharma Handelsgesellschaft GmbH, Burgdorf, Germany

${ }^{4}$ Isofluran $\mathrm{CP}^{\oplus}$ : CP-Pharma Handelsgesellschaft GmbH, Burgdorf, Germany

${ }^{5}$ Fentanyl-Janssen ${ }^{\bullet} 0.05$ mg/mL: Janssen-Cilag GmbH, Neuss, Germany

${ }^{6}$ Xylocain ${ }^{\circledast} 2$ \%: AstraZeneca GmbH, Wedel, Germany

${ }^{7}$ Ketamin 10 \%: Selectavet Dr. Otto Fischer GmbH, Weyarn-Holzolling, Germany

${ }^{8}$ Bupivacain-RPR-actavis ${ }^{\odot} 0.5 \%$ : Actavis Deutschland GmbH \& Co. KG, Langenfeld, Germany

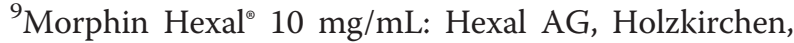
Germany

${ }^{10}$ Xylocain ${ }^{\oplus} 2$ \%, AstraZeneca GmbH, Wedel, Germany

${ }^{11}$ Rimadyl $^{\oplus}$ Injektionslösung: Pfizer $\mathrm{GmbH}$, Berlin, Germany

${ }^{12}$ Treadmill model 4060-80: Bertec Corporation, Columbus, OH, USA

${ }^{13}$ Vicon Motion Systems Ltd., Oxford, UK

${ }^{14} \mathrm{MX} 3+$ camera system: Vicon Motion Systems Ltd., Oxford, UK

${ }^{15}$ Vicon Calibration Device: Vicon Motion Systems Ltd., Oxford, UK

${ }^{16}$ Vicon Nexus: Vicon Motion Systems Ltd., Oxford, UK

${ }^{17}$ MyoResearch XP Master Edition, Noraxon U.S.A. Inc., Scottsdale, AZ, USA

${ }^{18}$ Microsoft $^{\bullet}$ Excel 2007

${ }^{19}$ Vicon Nexus and Bodybuilder: Vicon Motion Systems Ltd., Oxford, UK

${ }^{20}$ Philips Achieva 3.0 T X-series MRI: Philips Healthcare, Hamburg, Germany

${ }^{21}$ EIZO RadiForce ${ }^{\mathrm{TM}}$ RX211 Medical color LCD monitor: Enzo Nanao Corporation, Hakusan, Ishikawa, Japan

${ }^{22}$ GraphPad Prism ${ }^{\bullet}$ Version 4: GraphPad Software, Inc. La Jolla, California, USA

\section{Additional files}

Additional file 1: Video 1. Thirty-two kilogram patient walking on the treadmill at $0.8 \mathrm{~m} / \mathrm{s}$. Although the walking pattern is very similar between sessions, evident differences can be observed. (MPG $9336 \mathrm{~kb}$ )

Additional file 2: Video 2. Forty-kilogram dog (patient 7) walking and running 4 months after the amputation. Note that the dog has no difficulty in its movements whatsoever, neither when walking nor when running. (MPG $9560 \mathrm{~kb}$ )

Competing interests

The authors declare that no competing interests exist.

Authors' contributions

Conceived the study: IN. Designed the study: VGZ, VvB, NE, DB, IN, PW. Performed data collection: VGZ, VVB, NE. Analyzed the data: VGZ, PW. Wrote the paper: VGZ. Critical revision of the manuscript: VGZ, VvB, NE, DB, IN, PW. Final approval of the version to be published: VGZ, VvB, NE, DB, IN, PW.

\section{Acknowledgements}

The authors wish to thank Peter Dziallas, Beate Länger and Davina Wolf for performing the MRs, Martin Beyerbach for his statistical assistance and the patients' owners for their cooperation.

Partially funded by the National University of Colombia and the Colombian government in cooperation with the German Academic Exchange Service (DAAD) by a research scholarship awarded to VGZ.

\section{Author details}

${ }^{1}$ Small Animal Hospital, University of Veterinary Medicine Hannover, Foundation, Bünteweg 9, D-30559 Hannover, Germany. ${ }^{2}$ Small Animal Clinic, Faculty of Veterinary Medicine, National University of Colombia, Carrera 30 \# 45-03 (Ciudad Universitaria), Bogotá, Colombia.

Received: 24 December 2015 Accepted: 14 January 2016

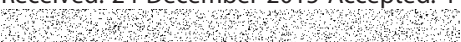




\section{References}

1. Stone EA. Amputation. In: Newton CD, Nunamaker DM, editors. Textbook of small animal orthopaedics. New York: IVIS Ithaca; 1985. p. 577-88.

2. Weigel JP. Amputations. In: Slatter DH, editor. Textbook of small animal surgery Saunders. Philadelphia: Saunders; 2003. p. 2180-90.

3. Lipowitz AJ. Complications in small animal surgery: diagnosis, management, prevention. Media: Williams \& Wilkins; 1996. p. 606-609.

4. Carberry CA, Harvey HJ. Owner Satisfaction with Limb Amputation in Dogs and Cats. J Am Anim Hosp Assoc. 1987;23:227-32.

5. Withrow SJ, Hirsch VM. Owner response to amputation of a pet's leg. Vet Med Small Anim Clin. 1979;74:332. 334.

6. von Werthern J, Horst C, Schwartz G. Zur Gliedmaßenamputaiton bei Hund und Katze: Eine Besitzerbefragung. Kleintierpraxis. 1999;44:169-76.

7. Kirpensteijn J, van den Bos R, Endenburg N. Adaptation of dogs to the amputation of a limb and their owners' satisfaction with the procedure. Vet Rec. 1999;144:115-8

8. Schulz M. Coping psychologically with amputation. Vasa. 2009;38 Suppl 74:72-4

9. Kirpensteijn J, van den Bos R, van den Brom WE, Hazewinkel HAW. Ground reaction force analysis of large breed dogs when walking after the amputation of a limb. Vet Rec. 2000;146:155-59.

10. Böddeker J, Drüen S, Meyer-Lindenberg A, Fehr M, Nolte I, Wefstaedt P. Computer-assisted gait analysis of the dog: Comparison of two surgical techniques for the ruptured cranial cruciate ligament. Vet Comp Orthop Traumatol. 2012;25:11-21.

11. Hottinger HA, DeCamp CE, Olivier NB, Hauptman JG, Soutas-Little RW. Noninvasive kinematic analysis of the walk in healthy large-breed dogs. Am J Vet Res. 1996;57:381-8.

12. Steiss JE, Yuill GT, White NA, Bowen JM. Modifications of a force plate system for equine gait analysis. Am J Vet Res. 1982;43:538-40.

13. Hielm-Bjorkman AK, Kuusela E, Liman A, Markkola A, Saarto E, Huttunen P, et al. Evaluation of methods for assessment of pain associated with chronic osteoarthritis in dogs. J Am Vet Med Assoc. 2003;222:1552-8.

14. Baird DK, Hathcock JT, Rumph PF, Kincaid SA, Visco DM. Low-field magnetic resonance imaging of the canine stifle joint: normal anatomy. Vet Radiol Ultrasound. 1998;39:87-97.

15. D'Anjou MA, Moreau M, Troncy E, Martel-Pelletier J, Abram F, Raynauld JP, et al. Osteophytosis, subchondral bone sclerosis, joint effusion and soft tissue thickening in canine experimental stifle osteoarthritis: Comparison between 1.5T magnetic resonance imaging and computed radiography. Vet Surg. 2008;37:166-77.

16. Blond L, Thrall DE, Roe SC, Chailleux N, Robertson ID. Diagnostic accuracy of magnetic resonance imaging for meniscal tears in dogs affected with naturally occuring cranial cruciate ligament rupture. Vet Radiol Ultrasound. 2008:49:425-31.

17. Boileau C, Martel-Pelletier J, Abram F, Raynould J-P, Troncy E, D'Anjou M-AD, et al. Magnetic resonance imaging can accurately assess the long-term progression of knee structural changes in experimental dog osteoarthritis. Ann Rheum Dis. 2008:67:926-32.

18. Gavin PR, Holmes SP. Orthopedic. In: Practical small animal MRI. Gavin PR, Bagley RS, editors. Ames: John Wiley \& Sons; 2009. p. 233-272.

19. Winegardner KR, Scrivani PV, Krotscheck U, Todhunter RJ. Magnetic resonance imaging of subarticular bone marrow lesions in dogs with stifle lameness. Vet Radiol Ultrasound. 2007:48:312-17.

20. Maes LD, Herbin M, Hackert R, Bels VL, Abourachid A. Steady locomotion in dogs: temporal and associated spatial coordination patterns and the effect of speed. J Exp Biol. 2008;211(Pt 1):138-49.

21. Riggs CM, DeCamp CE, Soutas-Little RW, Braden TD, Richter MA. Effects of subject velocity on force plate-measured ground reaction forces in healthy greyhounds at the trot. Am J Vet Res. 1993;54:1523-6.

22. Kim SY, Kim JY, Hayashi K, Kapatkin AS. Skin movement during the kinematic analysis of the canine pelvic limb. Vet Comp Orthop Traumatol. 2011:24:326-32.

23. Yam PS, Penpraze V, Young D, Todd MS, Cloney AD, Houston-Callaghan KA, et al. Validity, practical utility and reliability of Actigraph accelerometry for the measurement of habitual physical activity in dogs. J Small Anim Pract. 2011:52:86-91.

24. Bockstahler B, Muller M, Henninger W, Mayrhofer E, Peham C, Podbregar I. Kinetic and kinematic motion analysis of the forelimbs in sound Malinois dogs - sampling of basic values. Wien Tierarztl Monatsschr. 2008;95:127-38.

\section{Submit your next manuscript to BioMed Central and we will help you at every step:}

- We accept pre-submission inquiries

- Our selector tool helps you to find the most relevant journal

- We provide round the clock customer support

- Convenient online submission

- Thorough peer review

- Inclusion in PubMed and all major indexing services

- Maximum visibility for your research

Submit your manuscript at www.biomedcentral.com/submit
Biomed Central 